\title{
Infinite Length Results for Channel Shortening Equalizers
}

\author{
Richard K. Martin, C. Richard Johnson, Jr., Ming Ding, and Brian L. Evans \\ Richard K. Martin and C. Richard Johnson, Jr. \\ Cornell University \\ School of Electrical \& Computer Engineering \\ Ithaca, NY 14853, USA \\ \{frodo, johnson\}@ece.cornell.edu \\ Ming Ding and Brian L. Evans \\ The University of Texas at Austin \\ Dept. of Electrical \& Computer Engineering \\ Austin, TX 78712-1084, USA \\ \{ming, bevans\}eece.utexas.edu
}

Copyright 2003 IEEE. Published in the IV IEEE Signal Processing Workshop on Signal Processing Advances in Wireless Communications (SPAWC 2003), scheduled for June 15-18, 2003 in Rome, Italy. Personal use of this material is permitted. However, permission to reprint/republish this material for advertising or promotional purposes or for creating new collective works for resale or redistribution to servers or lists, or to reuse any copyrighted component of this work in other works, must be obtained from the IEEE. Contact:

Manager, Copyrights and Permissions

IEEE Service Center

445 Hoes Lane

P.O. Box 1331

Piscataway, NJ 08855-1331, USA.

Telephone: + Intl. 908-562-3966. 


\section{INFINITE LENGTH RESULTS FOR CHANNEL SHORTENING EQUALIZERS}

\author{
R. K. Martin and C. R. Johnson, Jr.* \\ Cornell University \\ School of Elec. and Comp. Engineering \\ Ithaca, NY 14853
}

\author{
M. Ding and B. L. Evans ${ }^{\dagger}$ \\ The University of Texas at Austin \\ Dept. of Elec. and Comp. Engineering \\ Austin, TX 78712-1084
}

\begin{abstract}
Time-domain equalization is crucial in reducing state dimension in maximum likelihood sequence estimation, and inter-carrier and inter-symbol interference in 802.11a and ADSL multicarrier systems. A time-domain equalizer, or TEQ, which is a finite impulse response (FIR) filter, placed in cascade with the channel produces an effective impulse response of $\nu+1$ samples that is shorter than the channel impulse response. This paper analyzes the two families of TEQ design methods amenable to cost-effective real-time implementation: minimum mean squared error (MMSE) and maximum shortening SNR (MSSNR) methods. For infinite length TEQs, we prove that MMSE target impulse responses are symmetric and have all $\nu$ zeros on the unit circle, and MSSNR TEQs have $\nu$ of their zeros on the unit circle. Consequently, finite-length MMSE and MSSNR TEQs will eventually yield increasing bit error rates (for broadcast systems) or decreasing bit rates (for point-to-point systems that allow bit allocation) with increasing filter length.
\end{abstract}

\section{INTRODUCTION}

Multicarrier modulation (MCM) techniques such as orthogonal frequency division multiplexing (OFDM) and discrete multi-tone (DMT) have been receiving increasing attention in the literature recently, and they have been deployed in numerous industry standards. Applications include the wireless LAN standards IEEE 802.11a and HIPERLAN2; Digital Audio Broadcast (DAB) and Digital Video Broadcast (DVB) in Europe; and asymmetric and very-high-speed digital subscriber loops (ADSL, VDSL). MCM is attractive due to the ease with which it can combat channel dispersion, provided that the channel delay spread is not greater than the length of the cyclic prefix (CP). The cyclic prefix is a copy of the last $\nu$ samples of each symbol which is prepended to the start of each symbol in order to make the convolution of

\footnotetext{
* This work was supported in part by NxtWave Communications (now ATI), Langhorne, PA.

${ }^{\dagger}$ This work was supported in part by The State of Texas Advanced Technology Program under project 003658-0614-2001.
}

the data and channel appear periodic. However, if the $\mathrm{CP}$ is not long enough, the orthogonality of the sub-carriers is lost and this causes both inter-carrier interference (ICI) and inter-symbol interference (ISI).

A well-known technique to combat the ICI/ISI caused by the inadequate $\mathrm{CP}$ length is the use of a time-domain equalizer (TEQ) in the receiver front end. The TEQ is a finite impulse response filter that shortens the channel so that the delay spread of the combined channel-equalizer impulse response is not longer than the CP length. The TEQ design problem has been extensively studied in the literature [1] - [12]. In [1], Falconer and Magee proposed a minimum-mean-square-error (MMSE) method for channel shortening, which was designed to reduce the complexity in maximum likelihood sequence estimation. More recently, Melsa, Younce, and Rohrs [5] proposed the maximum shortening SNR (MSSNR) method, which attempts to minimize the energy outside the window of interest while holding the energy inside fixed. This approach was generalized to the min-ISI method in [8], which allows the residual ISI to be shaped in the frequency domain. A blind, adaptive algorithm that searches for the TEQ maximizing the SSNR cost function was proposed in [10].

In point-to-point systems, the true performance measure to optimize is the maximum bit allocation that does not cause the error probability to exceed a threshold. In broadcast systems, the true performance measure is the bit error rate for a fixed bit allocation. Optimizing the MSE or SSNR does not necessarily optimize the bit rate [3], [7], [8], [11], [12] or the bit error rate.

This paper analyzes two families of TEQ design methods amenable to cost-effective real-time implementation: the MMSE and MSSNR designs. We show that the finite-length target impulse response corresponding to an infinite length MMSE TEQ is symmetric and has all $\nu$ of its zeros on the unit circle, and that the infinite length MSSNR TEQ has its $\nu$ dominant zeros on the unit circle. Our main contributions are generalizing these results from the results in [1], which assume white noise, zero delay, and a continuoustime TEQ (none of which would be expected to hold in practice); and demonstrating how rapidly the infinite length 
Table 1. Channel shortening notation

\begin{tabular}{|l|l|}
\hline Notation & Meaning \\
\hline \hline$x(k)$ & transmitted signal (IFFT output) \\
\hline$n(k)$ & channel noise \\
\hline$r(k)$ & received signal \\
\hline$y(k)$ & signal after TEQ \\
\hline$N$ & size of FFT \\
\hline$\nu$ & cyclic prefix (CP) length \\
\hline$\Delta$ & delay of effective channel \\
\hline $\mathbf{h}=\left[h_{0}, \cdots, h_{L_{h}}\right]$ & channel impulse response \\
\hline $\mathbf{w}=\left[w_{0}, \cdots, w_{L_{w}}\right]$ & TEQ impulse response \\
\hline $\mathbf{c}=\left[c_{0}, \cdots, c_{L_{c}}\right]$ & effective channel $(\mathbf{c}=\mathbf{h} \star \mathbf{w})$ \\
\hline $\mathbf{b}=\left[b_{0}, \cdots, b_{\nu}\right]$ & target impulse response \\
\hline$\tilde{L}_{h}=L_{h}+1$ & channel length \\
\hline$\tilde{L}_{w}=L_{w}+1$ & TEQ length \\
\hline$\tilde{L}_{c}=L_{c}+1$ & length of the effective channel \\
\hline $\mathbf{H}$ & $\tilde{L}_{c} \times \tilde{L}_{w}$ channel convolution matrix \\
\hline$\Omega=[\mathbf{0}, \mathbf{I}, \mathbf{0}]$ & row decimation matrix, see $(3)$ \\
\hline $\mathbf{R}_{x}$ & autocorrelation matrix of $x(k)$ \\
\hline $\mathbf{R}_{x r}$ & cross-correlation of $x(k)$ and $r(k)$ \\
\hline $\mathbf{I}_{N}$ & $N \times N$ identity matrix \\
\hline $\mathbf{A}^{*}, \mathbf{A}^{T}, \mathbf{A}^{H}$ & conjugate, transpose, and Hermitian \\
\hline
\end{tabular}

results are acheived by finite length filters.

The remainder of this paper is organized as follows. Section 2 reviews the multicarrier system model and notation. Section 3 derives the infinite length results. Section 4 shows, via simulations, how quickly the finite length filters approach the infinite length results, and Section 5 concludes the paper.

\section{SYSTEM MODEL AND NOTATION}

The multicarrier system model is shown in Fig. 1, and the notation is summarized in Table 1. Each block of bits is divided up into $N$ bins, and each bin is viewed as a QAM signal that will be modulated by a different carrier. An efficient means of implementing the multicarrier modulation in discrete time is to use an inverse fast Fourier transform (IFFT). The IFFT converts each bin (which acts as one of the frequency components) into a time-domain signal. After transmission, the receiver can use an FFT to recover the data within a bit error rate tolerance, provided that equalization has been performed properly.

In order for the subcarriers to be independent, the convolution of the signal and the channel must be a circular convolution. It is actually a linear convolution, so it is made to appear circular by adding a cyclic prefix to the start of each data block. The cyclic prefix is obtained by prepending the last $\nu$ samples of each block to the beginning of the block. If the $\mathrm{CP}$ is at least as long as the channel, then the output of each subchannel is equal to the input times a scalar complex gain factor. The signals in the bins can then be equalized by a bank of complex gains, referred to as a frequency domain equalizer (FEQ).

The above discussion assumes that $\mathrm{CP}$ length +1 is greater than or equal to the channel length. However, transmitting the cyclic prefix wastes time slots that could be used to transmit data. Thus, the CP is usually set to a reasonably small value, and a TEQ is employed to shorten the channel to this length. As discussed in Section 1, TEQ design methods have been well explored [1] - [12].

\section{INFINITE LENGTH RESULTS}

This section considers infinite length MMSE and MSSNR TEQ designs. Specifically, the goal is to show that in the limit, the target impulse response (TIR) becomes symmetric, with all of its zeroes on the unit circle.

Recall that the MMSE TEQ design uses a TEQ w and a TIR $b$ that must satisfy the relation [4]

$$
\mathbf{R}_{r x} \mathbf{b}=\mathbf{R}_{r} \mathbf{w}
$$

where $\mathbf{R}_{r x}$ is the channel input-output cross-correlation matrix and $\mathbf{R}_{r}$ is the channel output autocorrelation matrix. Typically, $\mathbf{b}$ is computed first, and then (1) is used to determine $\mathbf{w}$. The goal is that $\mathbf{h} \star \mathbf{w}$ approximates a delayed version of $\mathbf{b}$. As such, if $\mathbf{b}$ has nulls in its magnitude response, then w (or possibly h) will have them as well.

For an i.i.d. channel input sequence, the target impulse response is the eigenvector corresponding to the minimum eigenvalue of the $(\nu+1) \times(\nu+1)$ symmetric matrix [1], [4], [7]

$$
\begin{aligned}
\mathbf{R}_{\Delta} & =\mathbf{R}_{x}-\mathbf{R}_{x r} \mathbf{R}_{r}^{-1} \mathbf{R}_{r x} \\
& =\mathbf{I}_{(\nu+1)}-\Omega \mathbf{H}\left(\mathbf{H}^{T} \mathbf{H}+\mathbf{R}_{n}\right)^{-1} \mathbf{H}^{T} \Omega^{T},
\end{aligned}
$$

where $\mathbf{H}$ is the channel convolution matrix of size $\tilde{L}_{c} \times \tilde{L}_{w}$, and

$$
\Omega=\left[\mathbf{0}_{(\nu+1) \times \Delta}, \mathbf{I}_{\nu+1}, \mathbf{0}_{(\nu+1) \times\left(L_{c}-\nu-\Delta\right)}\right] .
$$

Robinson ([13], pp. 269-272) has shown that the eigenvector corresponding to the largest eigenvalue of a symmetric Toeplitz matrix will have all of its zeros on the unit circle, and Makhoul [14] has generalized this to show that the zeros of the eigenvector corresponding to the smallest eigenvalue has all of its zeros on the unit circle. (The proofs rely on the assumption that the corresponding eigenvalue has multiplicity 1.) Thus, we would like to show that in the limit of long TEQs, $\mathbf{R}_{\Delta}$ in (2) is Toeplitz (and clearly symmetric), which would imply that the MMSE TIR has its zeros on the unit circle. Furthermore, if we remove the term $\mathbf{R}_{n}$ from (2) and compute $\mathbf{b}$, then we obtain a windowed version of the MSSNR TEQ. So, this approach can also be used to show that the MSSNR TEQ has its dominant zeros on the unit circle. 


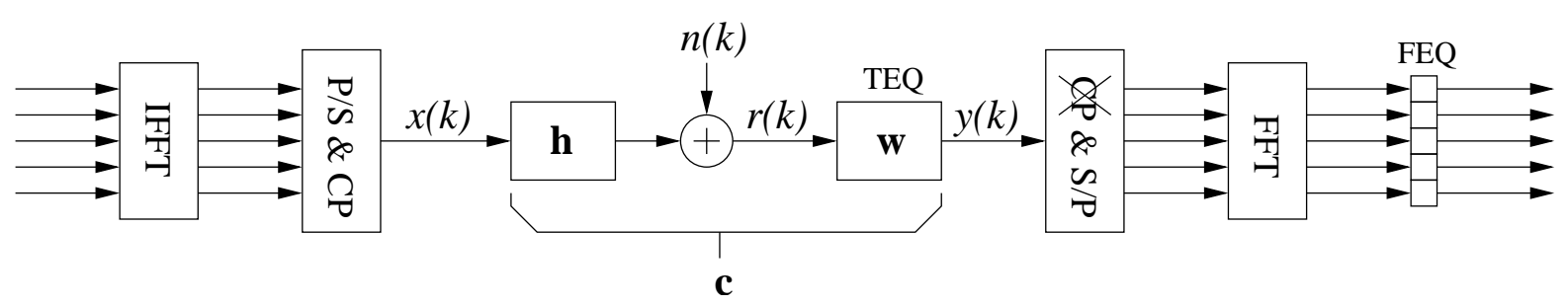

Fig. 1. Traditional multicarrier system model. (I)FFT: (inverse) fast Fourier transform, P/S: parallel to serial, S/P: serial to parallel, CP: add cyclic prefix, and XCP: remove cyclic prefix.

Theorem 3.1 Assume the input signal $x(k)$ is white, and the noise is non-zero (and possibly non-white). If the TEQ $\mathrm{w}$ is allowed to be any infinite length discrete-time filter, and if the minimum eigenvalue of $\mathbf{R}_{\Delta}$ has multiplicity 1 , then the finite length MMSE TIR $\mathbf{b}$ will be symmetric and will have all $\nu$ of its zeros on the unit circle. As a consequence, the effective channel impulse response $\mathbf{c}$ will have $\nu$ zeroes on the unit circle.

Proof: The proof loosely follows the less general proof in [1]. The first step is to show that $\mathbf{R}_{\Delta}$ becomes Toeplitz in the limit. For a white input, equation (1) can be rewritten as

$$
\mathbf{R}_{n} \mathbf{w}=\mathbf{H}^{T} \Omega^{T} \mathbf{b}-\mathbf{H}^{T} \mathbf{H} \mathbf{w} .
$$

Allowing $-\infty<i<\infty$, the $i^{\text {th }}$ component becomes

$$
\begin{aligned}
\sum_{j} R_{n}(i, j) w(j) & \\
= & \sum_{j=0}^{\nu} h(\Delta+j-i) b(j)-\sum_{j, l} h(l-i) h(l-j) w(j) \\
& =\sum_{j=0}^{\nu} h(\Delta+j-i) b(j)-\sum_{j} \phi(i-j) w(j),
\end{aligned}
$$

where $\phi(m)=\sum_{l} h(l) h(l+m)$ is the channel covariance function. In convolution notation,

$$
w(i) \star R_{n}(i)=b(i) \star h(\Delta-i)-w(i) \star \phi(i),
$$

where $R_{n}(m)$ is the noise autocorrelation function with ztransform $S_{n}(z)$. Taking z-transforms in (5),

$$
W(z) S_{n}(z)=B(z) z^{-\Delta} H\left(z^{-1}\right)-W(z) \Phi(z) .
$$

Solving for $W(z)$,

$$
W(z)=\frac{z^{-\Delta} B(z) H\left(z^{-1}\right)}{S_{n}(z)+\Phi(z)}
$$

We will make use of this equation shortly.

The error between the TEQ output and TIR output is

$$
e(k)=\sum_{l} b(l) x(k-\Delta-l)-\sum_{l} w(l) r(k-l) .
$$

The error covariance is

$$
\begin{aligned}
R_{e}(m) & \triangleq \mathrm{E}[e(k) e(k+m)] \\
=\mathrm{E} & {\left[\sum_{l_{1}, l_{2}} b\left(l_{1}\right) b\left(l_{2}\right) x\left(k-\Delta-l_{1}\right) x\left(k+m-\Delta-l_{2}\right)\right.} \\
& -\sum_{l_{1}, l_{2}} w\left(l_{1}\right) b\left(l_{2}\right) r\left(k-l_{1}\right) x\left(k+m-\Delta-l_{2}\right) \\
& -\sum_{l_{1}, l_{2}} w\left(l_{1}\right) b\left(l_{2}\right) r\left(k+m-l_{1}\right) x\left(k-\Delta-l_{2}\right) \\
& \left.+\sum_{l_{1}, l_{2}} w\left(l_{1}\right) w\left(l_{2}\right) r\left(k-l_{1}\right) r\left(k+m-l_{2}\right)\right] .
\end{aligned}
$$

Since $r(k)=\sum_{j} h(j) x(k-j)+n(k)$, when $x(k)$ is white with unit variance we obtain

$$
\begin{aligned}
& R_{e}(m)= \\
& \quad \sum_{l} b(l) b(m+l)-\sum_{l_{1}, l_{2}} w\left(l_{1}\right) b\left(l_{2}\right) h\left(\Delta-m+l_{2}-l_{1}\right) \\
& \quad-\sum_{l_{1}, l_{2}} w\left(l_{1}\right) b\left(l_{2}\right) h\left(\Delta+m+l_{2}-l_{1}\right) \\
& \quad+\sum_{l_{1}, l_{2}} w\left(l_{1}\right) w\left(l_{2}\right)\left[\phi\left(m+l_{1}-l_{2}\right)+R_{n}\left(m+l_{1}-l_{2}\right)\right]
\end{aligned}
$$

In convolution notation,

$$
\begin{aligned}
R_{e}(m)= & b(m) \star b(-m) \\
& -b(m-\Delta) \star w(\Delta-m) \star h(\Delta-m) \\
& -b(-m-\Delta) \star w(\Delta+m) \star h(\Delta+m) \\
& +w(m) \star w(-m) \star\left[\phi(m)+R_{n}(m)\right] .
\end{aligned}
$$

Taking z-transforms,

$$
\begin{aligned}
S_{e}(z)= & B(z) B\left(z^{-1}\right)-z^{-3 \Delta} B(z) W\left(z^{-1}\right) H\left(z^{-1}\right) \\
& -z^{3 \Delta} B\left(z^{-1}\right) W(z) H(z) \\
& +W(z) W\left(z^{-1}\right)\left[\Phi(z)+S_{n}(z)\right] .
\end{aligned}
$$

Now insert (7) into (9). Noting that $\Phi(z)=H(z) H\left(z^{-1}\right)$, 
and simplifying considerably,

$$
S_{e}(z)=B(z) B\left(z^{-1}\right) \underbrace{\left[\frac{S_{n}(z)-\Phi(z)\left(z^{\Delta}-z^{-\Delta}\right)^{2}}{S_{n}(z)+\Phi(z)}\right]}_{G(z)} .
$$

To minimize the MSE, we must minimize $R_{e}(0)$. By setting $z=e^{j \omega}$, taking the inverse Fourier transform, and setting $m=0$, we find that

$$
\begin{aligned}
R_{e}(0) & =\frac{1}{2 \pi} \int_{-\pi}^{\pi} S_{e}\left(e^{j \omega}\right) d \omega \\
& =\frac{1}{2 \pi} \int_{-\pi}^{\pi}\left\|B\left(e^{j \omega}\right)\right\|^{2} G\left(e^{j \omega}\right) d \omega,
\end{aligned}
$$

where $B\left(e^{j \omega}\right)=\mathbf{b}^{T}\left[1, e^{j \omega}, \ldots, e^{j \omega \nu}\right]^{T}$. Thus,

$$
\begin{aligned}
R_{e}(0) & =\mathbf{b}^{T} \mathbf{R}_{\Delta} \mathbf{b} \\
{\left[\mathbf{R}_{\Delta}\right]_{m, n} } & =\frac{1}{2 \pi} \int_{-\pi}^{\pi} e^{j \omega(m-n)} G\left(e^{j \omega}\right) d \omega=g(m-n) .
\end{aligned}
$$

Since $S_{n}\left(e^{j \omega}\right)$ and $\Phi\left(e^{j \omega}\right)$ are even functions in $\omega, G\left(e^{j \omega}\right)$ is as well. Thus, $\left[\mathbf{R}_{\Delta}\right]_{m, n}=\left[\mathbf{R}_{\Delta}\right]_{n, m}$, so $\mathbf{R}_{\Delta}$ is a symmetric Toeplitz matrix.

The optimal TIR $\mathbf{b}$ is the eigenvector corresponding to the minimum eigenvalue of $\mathbf{R}_{\Delta}$. Since $\mathbf{R}_{\Delta}$ is symmetric and Toeplitz, it is symmetric and centro-symmetric, so its eigenvectors (including b) will all be symmetric or skewsymmetric [15]. Observations suggest that the eigenvector (i.e. b) corresponding to the minimum eigenvalue of $\mathbf{R}_{\Delta}$ is always symmetric, but that has only been proven for the special case of a tridiagonal matrix [15].

Robinson [13] and Makhoul [14] have shown that the eigenvector corresponding to the minimum eigenvalue of a symmetric Toeplitz matrix has all of its zeros on the unit circle, so long as the eigenvalue has multiplicity 1 . This implies that the TIR has $\nu$ zeros on the unit circle. Since the TEQ has infinite length, the effective channel will be a zero-padded version of the TIR. Hence, the channel-TEQ frequency response will have $\nu$ zeros on the unit circle.

Remarks: First, an outline of the proof of a special case of this theorem was given in [1], which required that the noise was white, that $\Delta=0$, and that $\mathbf{w}$ was a continuoustime filter. The fact that the TIR in that special case was either symmetric or skew-symmetric was pointed out in [16], and the fact that the TIR in that special case has its zeros on the unit circle was observed in a footnote in [17]. Second, Theorem 3.1 specifies non-zero noise, but this is only necessary in the case $\Delta=0$, as can be seen from the proof. Daly, Heneghan, and Fagan [6] have shown that in the noiseless case with a white input, the MMSE and MSSNR design methods produce identical TEQs. If we set the noise to zero, then the proof of Theorem 3.1 is still valid, so long as $\Delta \neq 0$ (examine (10) and (12)). Thus, we can infer that for an infinite length MSSNR TEQ, the TEQ transfer function will have $\nu$ zeros on the unit circle.

For multicarrier systems, if a null lies at one of the subchannel carrier frequencies, then no data can be transmitted in that subchannel. This is a severe problem [7]. For pointto-point systems that use bit allocation, no bits can be allocated to these subchannels, so the bit rate suffers. This has been observed in [8]. For broadcast systems, the bit error rate on these subchannels will become very large, though this may be mitigated somewhat by coding across frequencies.

When the symmetry is exploited, the TIR design can be implemented more efficiently. For a finite length TEQ, the TIR is only approximately symmetric. If we force the TIR to be exactly symmetric, the computation of $\mathbf{b}$ will be reduced from an eigendecomposition of a matrix of size $(\nu+1) \times(\nu+1)$ to an eigendecomposition of a matrix of size $\lceil(\nu+1) / 2\rceil \times\lceil(\nu+1) / 2\rceil$, decreasing the complexity by a factor of 4 (since $\mathbf{R}_{\Delta}$ is symmetric).

\section{FINITE LENGTH RESULTS}

In the finite length case, $\mathbf{R}_{\Delta}$ is symmetric, but not quite Toeplitz, so the zeros of its eigenvector $\mathbf{b}$ will not be precisely on the unit circle. Analytic results for this case are intractable, so we give empirical results. Fig. 2 plots the average distance of the zeros of the TIR to the unit circle. The distances were averaged over carrier serving area (CSA) test channels $1-8$, which are standard channel models for DSL and are available at [18]. DSL channels were used since the channel models are publicly available and are commonly used in the channel shortening literature. There are 32 curves, one for each of the $\nu$ zeroes of the TIR. Most of the zeros start at a distance of about 0.2 from the unit circle. For a length $32 \mathrm{TEQ}$, the zeros are clustered around a distance of 0.01 from the unit circle; and for a length 100 TEQ, the zeros are clustered around a distance of $10^{-4}$ from the unit circle. The asymptotic results agree with Theorem 3.1.

\section{CONCLUSIONS}

This paper has analyzed the minimum mean squared error (MMSE) and maximum shortening SNR (MSSNR) channel shortening designs. For infinite length TEQs, MMSE target impulse responses are symmetric and have all zeros on the unit circle, and MSSNR TEQs has $\nu$ zeroes on the unit circle. Hence, finite-length MMSE and MSSNR TEQs eventually yield decreasing bit rate or increasing bit error rate with increasing length. In addition, forcing the TIR to be symmetric reduces the cost of computing the TIR by a factor of 4. 


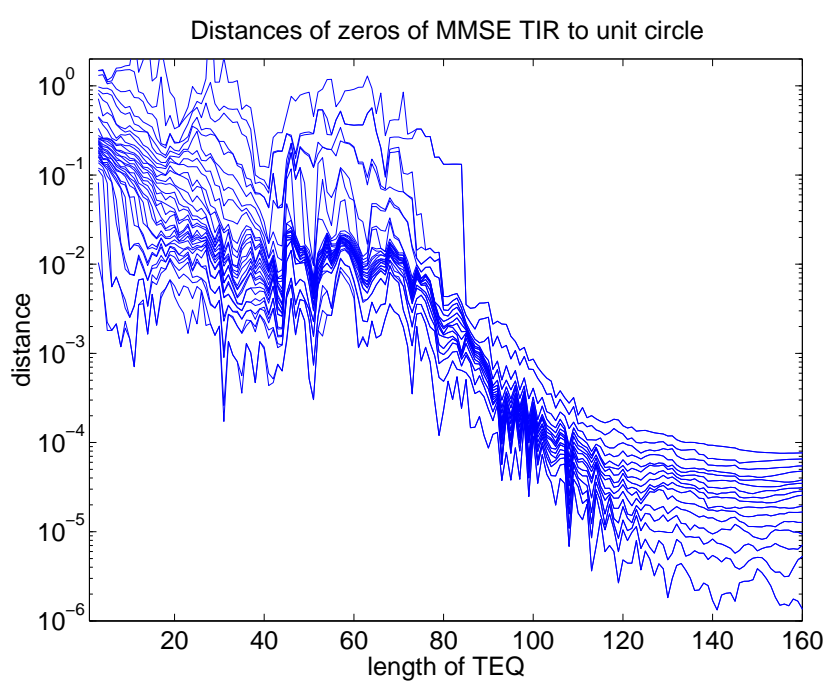

Fig. 2. Distance of the zeros of the MMSE TIR to the unit circle, averaged over CSA test channels 1 through 8. Each curve represents the distance for single zero.

\section{REFERENCES}

[1] D. D. Falconer and F. R. Magee, "Adaptive Channel Memory Truncation for Maximum Likelihood Sequence Estimation,” Bell Sys. Tech. Journal, pp. 15411562, Nov. 1973.

[2] J. S. Chow, J. M. Cioffi, and J. A. C. Bingham, "Equalizer Training Algorithms for Multicarrier Modulation Systems," in Proc. IEEE Int. Conf. on Comm., Geneva, Switzerland, May 1993, pp. 761-765.

[3] N. Al-Dhahir and J. M. Cioffi, "Optimum FiniteLength Equalization for Multicarrier Transceivers," IEEE Trans. on Comm., vol. 44, no. 1, pp. 56-64, Jan. 1996.

[4] N. Al-Dhahir and J. M. Cioffi, "Efficiently Computed Reduced-Parameter Input-Aided MMSE Equalizers for ML Detection: A Unified Approach," IEEE Trans. on Info. Theory, vol. 42, no. 3, pp. 903-915, May 1996.

[5] P. J. W. Melsa, R. C. Younce, and C. E. Rohrs, "Impulse Response Shortening for Discrete Multitone Transceivers," IEEE Trans. on Comm., vol. 44, pp. 1662-1672, Dec. 1996.

[6] D. Daly, C. Heneghan, and A. D. Fagan, "A Minimum Mean-Squared Error Interpretation of Residual ISI Channel Shortening for Discrete Multitone Transceivers," in Proc. IEEE Int. Conf. on Acoustics, Speech, and Signal Processing, May 2001, vol. 4, pp. 2065-2068.
[7] B. Farhang-Boroujeny and M. Ding, "Design Methods for Time-Domain Equalizers in DMT Transceivers," IEEE Trans. on Comm., vol. 49, no. 3, pp. 554-562, Mar. 2001.

[8] G. Arslan, B. L. Evans, and S. Kiaei, "Equalization for Discrete Multitone Receivers To Maximize Bit Rate," IEEE Trans. on Signal Processing, vol. 49, no. 12, pp. 3123-3135, Dec. 2001.

[9] N. Lashkarian and S. Kiaei, "Optimum Equalization of Multicarrier Systems: A Unified Geometric Approach," IEEE Trans. on Comm., vol. 49, pp. 17621769, Oct. 2001.

[10] R. K. Martin, J. Balakrishnan, W. A. Sethares, and C. R. Johnson, Jr., "A Blind, Adaptive TEQ for Multicarrier Systems," IEEE Signal Processing Letters, vol. 9, no. 11, pp. 341-343, Nov. 2002.

[11] M. Milosevic, L. F. C. Pessoa, B. L. Evans, and R. Baldick, "Optimal Time Domain Equalization Design for Maximizing Data Rate of Discrete Multi-tone Systems," IEEE Trans. on Signal Processing, under revision.

[12] K. Vanbleu, G. Ysebaert, G. Cuypers, M. Moonen, and K. Van Acker, "Bitrate Maximizing Time-Domain Equalizer Design for DMT-based Systems," IEEE Trans. on Comm., under revision.

[13] E. Robinson, Statistical Communication and Detection, Griffin, London, 1967.

[14] J. Makhoul, "On the Eigenvectors of Symmetric Toeplitz Matrices," IEEE Trans. on Acoustics, Speech, and Signal Processing, vol. 29, pp. 868-872, Aug. 1981.

[15] A. Cantoni and P. Butler, "Eigenvalues and Eigenvectors of Symmetric Centrosymmetric Matrices," Linear Algebra and Its Applications, vol. 13, pp. 275-288, 1976.

[16] A. Cantoni and P. Butler, "Properties of the Eigenvectors of Persymmetric Matrices with Applications to Communication Theory," IEEE Trans. on Comm., vol. 24, pp. 804-809, Aug. 1976.

[17] N. Al-Dhahir, Optimized-Transmitter ReducedComplexity MMSE-DFE under Finite-Length Constraints, Ph.D. thesis, Stanford University, 1994.

[18] G. Arslan, M. Ding, B. Lu, M. Milosevic, Z. Shen, and B. L. Evans, "MATLAB DMTTEQ Toolbox Version 3.1," The University of Texas at Austin, http://www.ece.utexas.edu/ bevans/projects/adsl/ dmtteq/dmtteq.html. 